\title{
Minimizing Time in Scheduling of Independent Tasks Using Distance-Based Pareto Genetic Algorithm Based on MapReduce Model
}

\author{
Devarajan Rajeswari*, Veerabadran Jawahar Senthilkumar \\ Department of Electronics and Communication Engineering, College of Engineering, Anna University, \\ Chennai, India \\ Email: *drajiit@gmail.com
}

Received 21 March 2016; accepted 9 May 2016; published 12 May 2016

Copyright (C) 2016 by authors and Scientific Research Publishing Inc.

This work is licensed under the Creative Commons Attribution International License (CC BY). http://creativecommons.org/licenses/by/4.0/

\section{(c) (i) Open Access}

\begin{abstract}
Distributed Systems (DS) have a collection of heterogeneous computing resources to process user tasks. Task scheduling in DS has become prime research case, not only due of finding an optimal schedule, but also because of the time taken to find the optimal schedule. The users of Ds services are more attentive about time to complete their task. Several algorithms are implemented to find the optimal schedule. Evolutionary kind of algorithms is one of the best, but the time taken to find the optimal schedule is more. This paper presents a distance-based Pareto genetic algorithm (DPGA) with the Map Reduce model for scheduling independent tasks in a DS environment. In DS, most of the task scheduling problem is formulated as multi-objective optimization problem. This paper aims to develop the optimal schedules by minimizing makespan and flow time simultaneously. The algorithm is tested on a set of benchmark instances. MapReduce model is used to parallelize the execution of DPGA automatically. Experimental results show that DPGA with MapReduce model achieves a reduction in makespan, mean flow time and execution time by $12 \%, 14 \%$ and $13 \%$ than non-dominated sorting genetic algorithm (NSGA-II) with MapReduce model is also implemented in this paper.
\end{abstract}

\section{Keywords}

Distributed Systems, Multi-Objective, MapReduce, Optimization, DPGA, NSGA-II

\footnotetext{
*Corresponding author.
}

How to cite this paper: Rajeswari, D. and Senthilkumar, V.J. (2016) Minimizing Time in Scheduling of Independent Tasks Using Distance-Based Pareto Genetic Algorithm Based on MapReduce Model. Circuits and Systems, 7, 735-747. 


\section{Introduction}

The Computational power of individual system is not sufficient for solving widely used complex computational tasks like high energy physics, earth science, etc. In order to solve complex jobs, high performance parallel and distributed systems are developed with a number of processors. As the computing nodes are heterogeneous in a multiprocessor environment, execution time of tasks varies on each processor. Scheduling of tasks is a key issue, to achieve the high usability of supercomputing capacity of distributed computing environment [1]. To ensure efficient utilization of resources, suitable scheduling algorithms are used to assign the tasks to the available processors efficiently.

For distributed computing environment, static scheduling can be used due to geographically distributed computing resources with various ownerships, access policy and different constraints. Schedulers are developed using complex arithmetic techniques that use the available values of application and environment. So, heuristic methods are the best approach to find the optimal schedule in the DS environment [2]. The most important criteria used to analyze the efficiency of scheduling techniques are makespan and flowtime. Time taken to complete the last task is Makespan [3] and the sum of completion time of all tasks in a schedule is flowtime. The schedule, which optimizes the makespan and flowtime is called as optimum schedule [4]. To minimize makespan, the Longest Job to be scheduled to Fastest Resource (LJFR) and for minimizing flowtime, the Shortest Job to be scheduled to Fastest Resource (LJFR) [2]. Flowtime minimization makes the makespan maximization. This leads the problem as multiple objective.

Genetic Algorithm (GA) is a search and population-based model [5]. This has been extensively used in various problem domains. GA has the capability to search various regions of the solution space and note a diverse set of solutions for the distributed computing problem. GA uses genetic operators to improve the structure of good solutions in various objective spaces. These characteristics of GA used to find the best optimal schedule for multi-objective problem in distributed systems. The distance-based Pareto genetic algorithm (DPGA) of Osyczka [6] is used in this paper. DPGA uses a distance computation and dominance test procedure and elitist method of combining parent population with the offspring population for the next iteration. Set of most difficult static benchmark instances of Braun et al. [1] is used to analyze the performance of NSGA-II. The degree of task and resource heterogeneity can be captured by these instances. Hadoop MapReduce is a framework for distributed large scale data processing on computer clusters. MapReduce is used to automatically parallelize the data processing on clusters in a reliable and fault-tolerant manner [7].

MapReduce programming model is used to implement the multi-objective DPGA to find the optimal schedule with minimum time in the distributed computing environment. The DPGA with MapReduce model suits for distributed computing environments with various computing resources and scheduling is done by considering makespan and flowtime minimization. The DPGA with MapReduce model generates better solutions in minimal time than NSGA-II with MapReduce model.

The remainder of the paper is structured as follows. Section 2 discusses the literature review. Multi-objective optimization introduction is presented in Section 3. Section 4 determines for identifying the variety of distributed computing environment in the simulation process. A Scheduling method using NSGA-II with MapReduce and DPGA with MapReduce is available in Section 5. Simulation results are shown in Section 6. Finally, Section 7 concludes and discusses the future work.

\section{Related Work}

Optimal scheduling of independent tasks to available resources in DS is an NP-complete problem and it depends on various heuristics and meta-heuristic algorithms. A few well known heuristic methods are min-max [8], suffrage [9], min-min, max-min [10] and LJFR-SJFR [11]. These above heuristic methods are more time consuming process. In recent, several meta-heuristic methods are developed to solve complex computational problems. The most popular methods are GA [6], particle swarm optimization (PSO) [12], ant colony optimization (ACO) [13] and simulated annealing (SA) [14]. The description of eleven heuristics and comparison on the various distributed environment was done by Braun et al. [1] and illustrates the effectiveness of GA with others. All the above meta-heuristic methods considered single objective and aimed to minimize the makespan.

There are some methods considered multiple objectives, while scheduling tasks in distributed environments. Izakian et al. [15] compares five heuristics depends on the machine, and task characteristics for minimizing both makespan and flowtime, but calculated separately. Several nature inspired meta-heuristic methods like GA, 
ACO and SA for scheduling tasks in a grid computing environment by using single and multi-objective optimization was done by Abraham et al. [16]. Xhafa et al. [17] implemented GA based scheduler. All the above methods convert the multi-objective optimization problem into a scalar cost function, which makes single objective before optimization.

To minimize the amount of time to find the best optimal schedule Lim et al. [18] implemented PGA, Durillo et al. [19] implemented parallel execution of NSGA-II and these methods have the difficulties to make communication and synchronization between the resources in a distributed environment. This paper implements NSGA-II with MapReduce programming model and DPGA with MapReduce programming model to find the best optimal schedule. It makes the task scheduling as an efficient real time multi-objective optimization problem.

\section{Multi-Objective Optimization}

The Scheduling problem in a distributed environment needs to optimize the several objectives at the same time. In general, these objectives are contradictory with each other. These contradictory objective functions generates a set of optimal solutions. In the optimal solution set, not one solution is greater than each another solution with regarding all the objective functions. These optimal solution set is called as Pareto optimal solution. The multiobjective minimization problem is formulated as,

$$
\text { Minimize } z=\left(g_{1}(a), g_{2}(a), \cdots, g_{m}(a)\right) \quad \text { subject to } a \in S
$$

where $a=\left[a_{1}, a_{2}, \cdots, a_{n}\right]$ is the vector of decision variables, $g_{k}: \mathfrak{R}^{n} \rightarrow \mathfrak{R}, k=1,2, \cdots, m$ is the objective functions and $S \subset \mathfrak{R}^{n}$ is the suitable region in the decision space. A result $a \in S$ is said to dominate another result $b \in S$, if the subsequent things are satisfied,

$$
\begin{array}{ll}
\forall k \in\{1,2, \cdots, m\}, & g_{k}(a) \leq g_{k}(b) \\
\exists k \in\{1,2, \cdots, m\}, & g_{k}(a)<g_{k}(b)
\end{array}
$$

$a$ is said to be a Pareto optimal solution, where no solution dominates $a \in S$. The best solution of the Pareto optimal set is called as a Pareto optimal front in multi objective problem space. Once the entire Pareto optimal solution is found, that is the indication of completing multi-objective problem [20].

Multi objective optimization is also known as vector optimization, as a vector of objectives is optimized instead of a single objective. When using multiple objectives, the search space is divided into two non overlapping regions known as optimal and non-optimal. The difference between single objective and multiple objective optimization are handling two search spaces and having two goals instead of single.

\section{Problem Statement}

Distributed environment has geographically distributed computing systems with complex combinations of hardware, software and network components. $R$ is the set of $\mathrm{m}$ processing elements in distributed environment and $\mathrm{T}$ is the set of $\mathrm{n}$ tasks assigned to the processing elements. As scheduling is performed for independent tasks, there is no communication among the tasks and a task can be assigned to a processing element exclusively. The pre-emption of task is not allowed. As scheduling is performed statically, computing capacity and prior load of processing element and computational load of the task is estimated and the tasks are scheduled in batches, once allocated the tasks it cannot be migrated to another resource. Expected time to compute matrix (ETC) can be built by using these details. An ETC matrix is a $\mathrm{p} \times \mathrm{q}$ matrix, in which each position of the matrix, ETC [p] [q] illustrates the expected time to complete job $\mathrm{p}$ in resource $\mathrm{q}$. The row of ETC matrix has the completion time of a job of each resource and each column specifies the estimated execution time of a resource for all the jobs. Hence, the proposed method is static, non-preemptive scheduling.

In this paper, the objective considered is minimization of makespan and flow time. Makespan is the completion time and waiting time of a task in a processing element. Flow time is defined as the sum of completion time of all the tasks described as follows,

$$
\text { makespan }=\min _{s_{j} \in S c h}\left\{\max _{t \in t a s k s} F_{t}\right\}
$$




$$
\text { flowtime }=\min _{s_{j} \in S c h}\left\{\sum_{t \in t a s k s} F_{t}\right\}
$$

where $F_{t}$ the completion time of task $t$, tasks stands for a set of all tasks, $S c h$ is set of all possible schedules. Longest job has to be scheduled on fastest resource to minimize the makespan and for minimizing flow time, shortest job to be scheduled on fastest resources. This contradiction makes the problem as multi-objective.

\section{Multi-Objective GA with MapReduce for Scheduling Tasks to the Distributed Environment}

\subsection{Elitist Non-Dominated Sorting Genetic Algorithm (NSGA-II)}

Initial populations of size $\mathrm{N}$ are generated randomly. Non-dominated sorting is performed on the population to classify it into a number of fronts. Crowded tournament selection is performed by assigning crowding distance. This is used to select a better ranked solution if they belong to different front or select the higher crowding distance solution if they belong to same front. Crossover and mutation are performed on the generated parent solution and produce the offspring with size N. Single point crossover and swap mutation is used. The parent and offspring population of size $\mathrm{N}$ are combined and produce $2 \mathrm{~N}$ population. Update the population by the individuals from lower front to size n. The individual has small crowding distance will be dropped in the tie. Precede again, except the first step till meets the stopping criteria [21]. Figure 1 shows the workflow of NSGA-II with MapReduce model.

Non-dominated sorting: It is used to find the individuals to the next iteration by classifying the population. The procedure is given below [22].

Step 1: For individual solution $\mathrm{p}$ in population $\mathrm{N}$.

Step 2: For individual solution $\mathrm{q}$ in population $\mathrm{N}$.

Step 3: If $\mathrm{p}$ and $\mathrm{q}$ are not equal, Compare $\mathrm{p}$ and $\mathrm{q}$ for all the objectives.

Step 4: For any $\mathrm{p}$, q is dominated by $\mathrm{p}$, mark solution $\mathrm{q}$ as dominated.

First non-dominated set is formed from unmarked solutions.

Step 5: Repeat the procedure till the entire population is divided into fronts.

Selection: The Crowded tournament selection operator is used. An individual i win the tournament with another individual $\mathrm{j}$, if one of the following is true [22].

1. An individual i have better rank, i.e., ranki $<$ rankj.

2. The individual $\mathrm{i}$ and $\mathrm{j}$ have the same rank (ranki $=$ rankj), then the individual $\mathrm{i}$ has better crowding distance (in less crowded areas, i.e., $\mathrm{di}=\mathrm{dj}$ ) than individual $\mathrm{j}$.

Crowding distance calculation: To break the tie between the individuals are having the same rank crowding distance is used [22]. The procedure is as follows,

Step 1: Initialize the number of individuals $(x)$ in the front $(\mathrm{Fa})$.

Step 2: Set the crowding distance $d_{i}=0, \quad i=1,2, \cdots, x$.

Step 3: Sort the individuals (x) in front (Fa) based on the objective function (obj). obj $=1,2, \cdots, m$. $m$ is the number of objectives and $S=$ sort (Fa, obj).

Step 4: Set the distance of boundary individuals as $S\left(d_{1}\right)=\infty$ and $S\left(d_{x}\right)=\infty$.

Step 5: Set $k=2$ to $(x-1)$ and calculate $S\left(d_{2}\right)=S\left(d_{x-1}\right)$ as follows

$$
S\left(d_{k}^{m}\right)=S\left(d_{k}\right)+\frac{S(k+1) f_{m}-S(k-1) f_{m}}{f_{m}^{\max }-f_{m}^{\min }}
$$

$S(k) f_{m}$ is the $k^{\text {th }}$ individual value in $S$ for $m^{\text {th }}$ objective function.

\subsection{Distance-Based Pareto Genetic Algorithm (DPGA)}

At first distance-based fitness assignment was proposed by Osyczka and Kundu [6], the idea giving importance to both Pareto optimal front and the diversity of that front in one fitness measure. DPGA uses a distance computation and dominance test procedure with complexity O (kn2) [4]. DPGA maintains a standard GA population and Elite population. The genetic operations like selection, crossover and mutation are performed on GA population. All the non-dominated solutions are maintained in elite population. The steps are as follows [23]:

Step 1: Initialize the random population of size N (P0) 


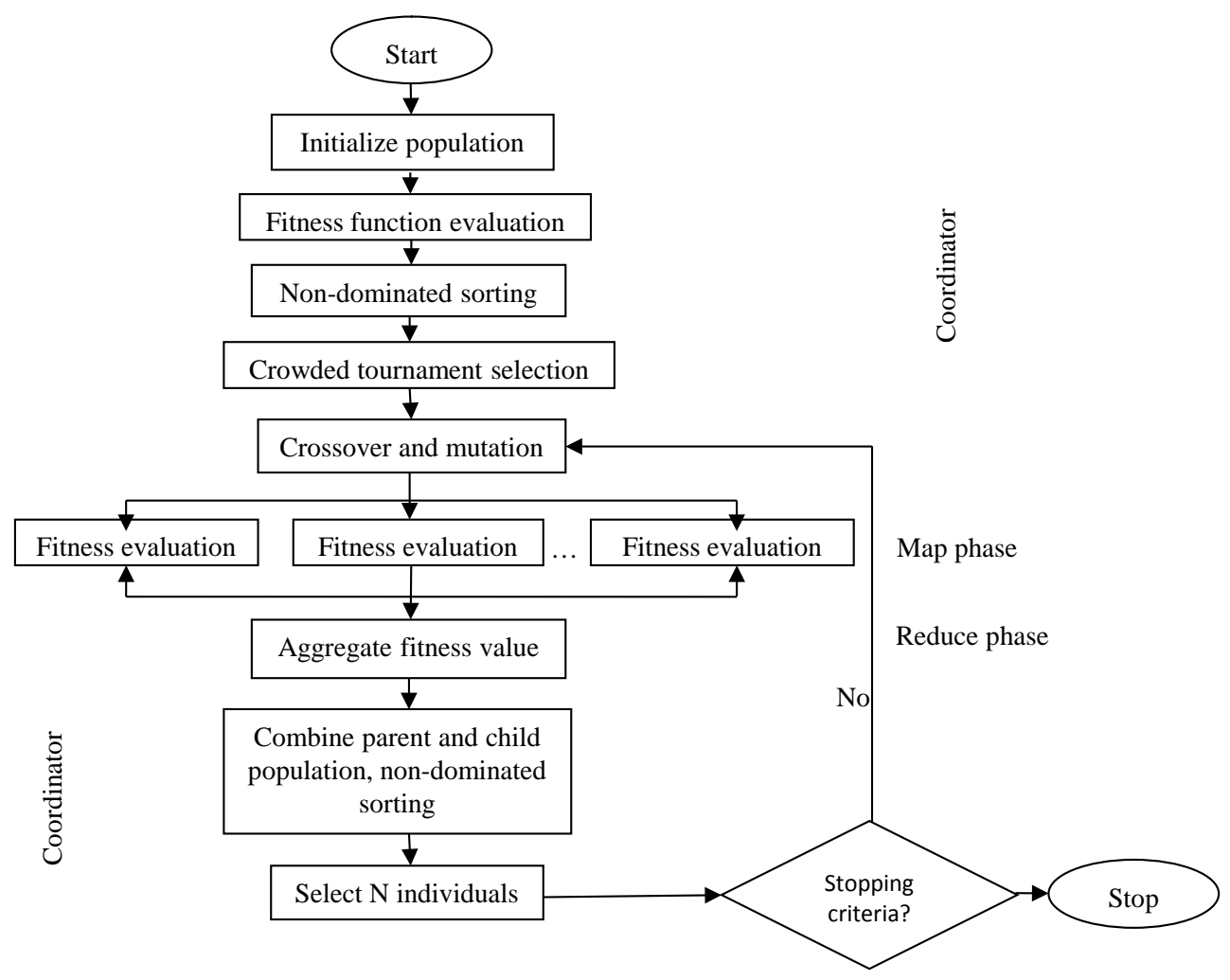

Figure 1. Flowchart for NSGA-II with MapReduce.

Step 2: Calculate the fitness for the first solution (F1) and set generation counter $\mathrm{c}=0$.

Step 3: If $\mathrm{c}=0$, insert the first element of $\mathrm{P} 0$ in the elite set $\mathrm{E} 0$ and perform the distance calculation, minimum distance, index of elite member, elite population updating for each member of the population $(k \geq 2)$ and $\mathrm{k} \geq 1$ for $\mathrm{c}>0$.

3a. Distance Calculation

Distance calculation is to find the distance between population member and elite member in the objective space [23]. The formula for calculating distance is given below:

$$
d_{k}^{i}=\sqrt{\sum_{m=1}^{M} \frac{e_{m}^{(i)}-f_{m}^{(k)}}{e_{m}^{(i)}}}
$$

$e_{m}$ is the fitness of elite member, $f_{m}$ is the fitness of population member for particular objectives.

3b. Minimum Distance Calculation

Find the minimum distance of a population member compared to all the member of an elite set [23]. It is calculated as follows,

$$
d_{k}^{\min }=\min _{i=1}^{\left|E_{c}\right|} d_{k}^{i}
$$

$E$ is an elite set, $d_{k}$ is the calculated distance.

3c. Elite member index [23].

The Elite member index is used to find which member of elite set has near to the member of the population

$$
i_{k}^{*}=\left\{i: d_{k}^{i}=d_{k}^{\min }\right\}
$$

$d_{k}^{i}$ is the calculated distance and $d_{k}^{\min }$ is the minimum distance.

3d. Fitness Calculation and Elite set update

The elite set is updated depends on the domination of population member [23]. Population member $(k)$ is 
dominated by elite solution, the fitness of $k$ is

$$
F_{k}=\max \left[0, F\left(e^{i_{k}^{*}}\right)-d^{\min }\right]
$$

Otherwise

$$
F_{k}=\left[F\left(e^{i_{k}^{*}}\right)+d^{\min }\right]
$$

and the population member is included by eliminating all dominated elite members in elite set.

Step 4: Find the minimum fitness value among all the members of elite population and all elite solutions are assigned fitness $F_{\min }$.

4a. Minimum fitness

Minimum fitness is used to find minimum fitness value among all the members of elite population and all elite solutions are assigned a fitness Fmin [23] is,

$$
F_{\text {min }}=\min _{i=1}^{\left|E_{c}\right|} F_{j}
$$

$E$ is elite set; $F$ is the fitness of elite solution.

Step 5: Stop c $+1=c_{\max }$ or termination criterion is satisfied. Otherwise, go to step 6 .

Step 6: Generate new population ( $\mathrm{Pc}+1)$ by using selection, crossover and mutation on Pc. Set $c=c+1$ and go to step 3.

There is no genetic operations like reproduction, crossover and mutation is performed on elite population Ec explicitly. To generate the new population selection, crossover and mutation operators are used. In this paper, tournament selection, single point crossover and swap mutation are used. Figure 2 shows the workflow of DPGA with MapReduce model.

\subsection{MapReduce Model}

Hadoop: Hadoop is an open source framework that provides reliable, scalable, distributed processing and storage on large clusters of inexpensive servers [24]. Hadoop is written in Java and users can customize the code to parallelize the data process in clusters which contains thousands of commodity servers. The response time

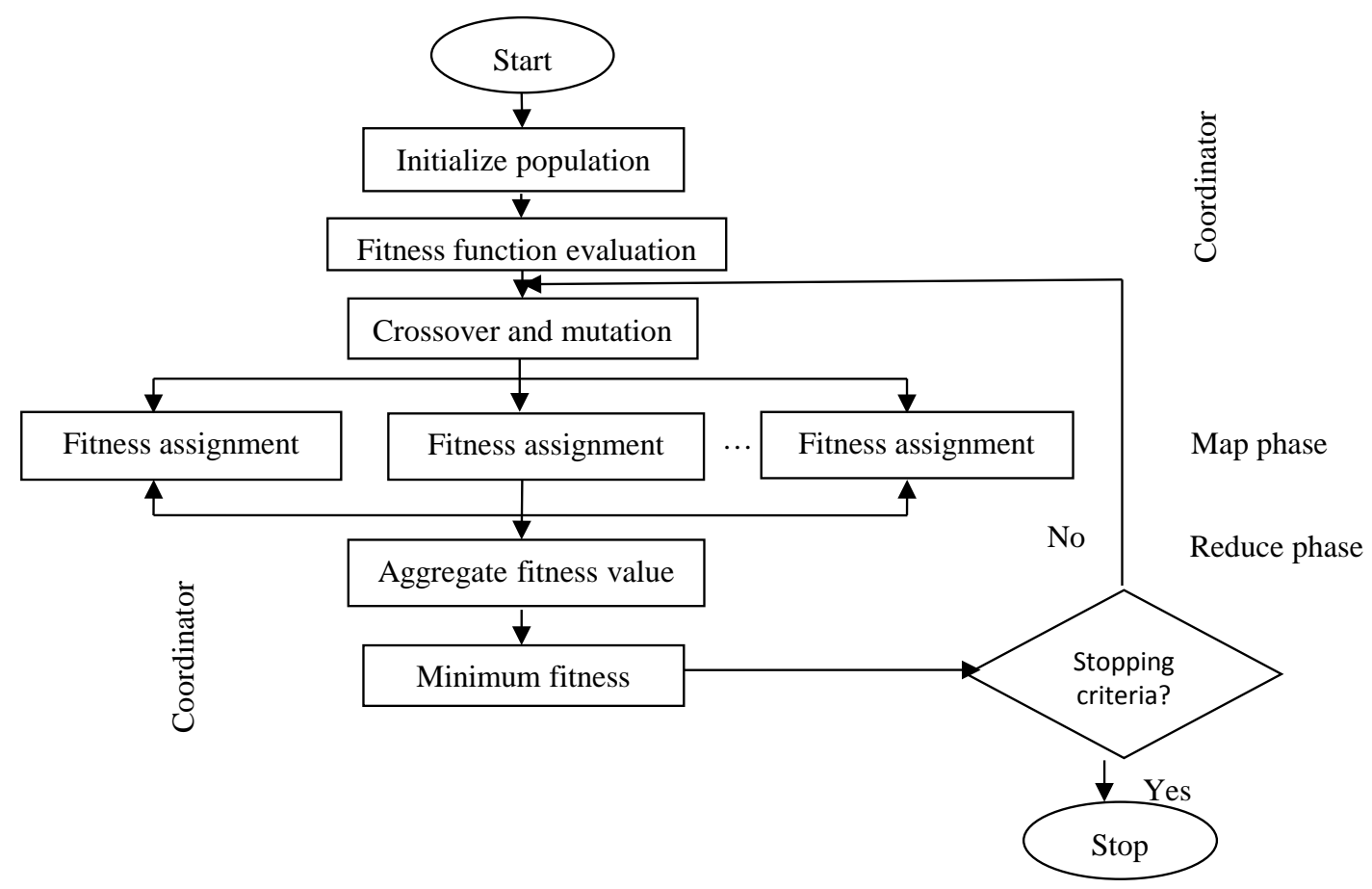


depends on the complexity of process and volumes of data. The advantage of Hadoop is its massive scalability. The Apache Hadoop framework consists of Hadoop kernel, MapReduce, Hadoop Distributed File System (HDFS) and some related projects like HBase, Hive and Zookeeper. At present, Hadoop plays a major role in Email spam detection, search engines, genome manipulation in life sciences, advertising, prediction in financial service and analysis of log files. Linux and Windows are a preferred operating system for Hadoop.

HDFS: A file system component of Hadoop is called as HDFS. Distributed low-cost hardware is used to store data in HDFS.HDFS contains name node and data node. A name node has Meta data information. If there is a request to read a data from HDFS, the name node provides the location of data blocks. The name node also has overall system information. So the name node is called as master of HDFS. The secondary name node has the replication of Meta data. At first, data node has to be registered in name node and gets namespace ID. For every particular period of time, the data node updates its status to name node. HDFS splits the large file into blocks and stored it in the different data nodes. Each block is replicated at the nodes of Hadoop cluster. At the time of failure data is re-replicated by the active Hadoop monitoring system [25].

MapReduce programming: It is a distributed parallel processing of large volume of data in a cluster with fault tolerant and reliable manner. MapReduce has job tracker and task tracker. Job tracker splits the job into tasks and schedules it to task tracker. The job tracker monitors the progress of task tracker. It is also responsible for re-executing the failed tasks. The map phase splits a user program into sub tasks and generates a set of key-value pairs. It will be submitted to reduce after a shuffle. The reduce phase performs user supplied reduce function on same key values to generate single entity. The reduce phase is also called as merge phase. The workflow of MapReduce is presented in Figure 3. The MapReduce function is represented as,

map:: (input__record) => list (key, values).

reduce:: (key, list (values) => key, aggregate (values).

\subsubsection{NSGA-II with MapReduce}

The fitness evaluation of offspring alone has done parallel in the workers available in the map phase. Non-dominated sorting, crowded tournament selection is performed on an entire population. So it cannot be fit into concurrent process. 1) Initial population is loaded into coordinator. 2) Coordinator evaluates the fitness value; perform non-dominated sorting, crowded tournament selection, crossover and mutation. The offspring generated by coordinator sends to job tracker. 3) The job tracker splits the offspring population and send to workers of map phase to evaluate fitness value in parallel manner and send it to reduce phase. 4) Shuffle operation is performed between map and reduce phase. 5) The workers of reduce phase aggregate the fitness value and send it to the coordinator.

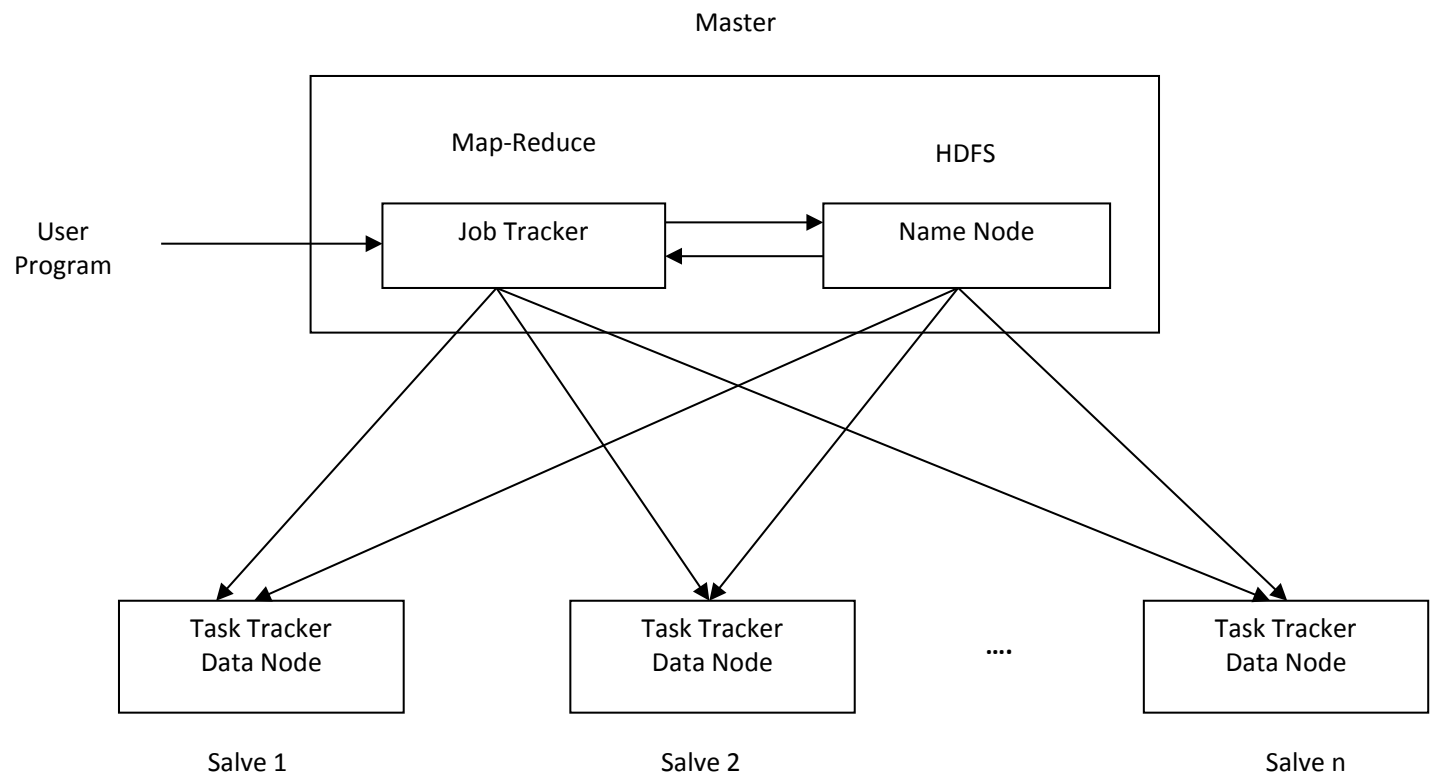

Figure 3. Workflow on MapReduce and HDFS. 


\subsubsection{DPGA with MapReduce}

This approach can parallelize the distance calculation, minimum distance calculation, elite member index and fitness calculation. The evaluation of individuals is executed parallel, because the fitness calculation is independent from others in a population. 1) The initial population is seeded into the coordinator 2) The coordinator produces the offspring population 3) The job tracker divides the offspring population into sub populations and assigns to workers in the map phase 4) The workers perform the distance calculation, minimum distance calculation, elite member index and fitness evaluation for assigned individuals concurrently 5) The workers of reduce phase collect the fitness values and perform merge operation then send it back to coordinator for the next generation.

\section{Simulation Results and Discussion}

The proposed DPGA with MapReduce model is carried out. To estimate the efficiency, NSGA-II with MapReduce model is also implemented. The metrics considered for performance evaluation are execution time, makespan and flowtime.

\subsection{Simulation Environment}

The simulation is designed by writing programs in Hadoop MapReduce using Java. Hadoop1.2.1 stable version is used to set up 4 node cluster, which is backed up by HDFS. Hadoop cluster is running on Ubuntu Linux platform and Java 1.6 is used for writing the code. All the 4 systems have i5 processors, 4GB RAM and 500GB hard disk. The proposed method is evaluated based on factors available in Table 1. Random generation with uniform distribution is used for simulation. Resources can execute a task at a time. ETC matrix is generated depends on three metrics: task and resource heterogeneity and consistency. The various instances are labeled as x-yy-zz that represented as follows,

x-consistency type (co-consistent, ic—inconsistent, sc—semi consistent).

yy-task heterogeneity (hi一-high, lo-low).

zz-processor heterogeneity (hi-high, lo-low).

\subsection{Result Discussions}

The algorithm DPGA with MapReduce and NSGA-II with MapReduce model apply to all 12 problem instances. To compare the performance of multi-objective scheduling algorithm in a distributed computing environment, the Pareto optimal solutions produced by the two methods are plotted in Figures 4-7 for all the instances. For a better comparison of both the algorithm, each was run 10 times repeatedly with various random seeds and the best solutions are considered for both DPGA with MapReduce and NSGA-II with MapReduce.

The makespan and mean flowtime are deliberate in same time units and obtained Pareto optimal solutions are plotted on a scale of ten thousands of time unit. The plotted graphs indicate that DPGA with MapReduce produces best schedule in terms of the minimization of makespan and flowtime for all cases compared to NSGA-II MapReduce method. The algorithms are run for 1000 iterations and 100 initial populations were taken. It is also noted that the number of solutions obtained in DPGA with MapReduce increases by increasing number of

\begin{tabular}{|ccc|}
\hline Table 1. Specification setting. & & \\
\hline Specifications & NSGA-II & DPGA \\
\hline Population size & 200 & 200 \\
Number of iteration & 1000 & 0.8 \\
Crossover probability $\left(\mathrm{p}_{\mathrm{c}}\right)$ & 0.8 & 0.01 \\
Mutation probability $\left(\mathrm{p}_{\mathrm{m}}\right)$ & 0.01 & Single point \\
Crossover type & Single point & Swap \\
Mutation type & Swap & Large tournament \\
Selection type & Crowded tournament
\end{tabular}




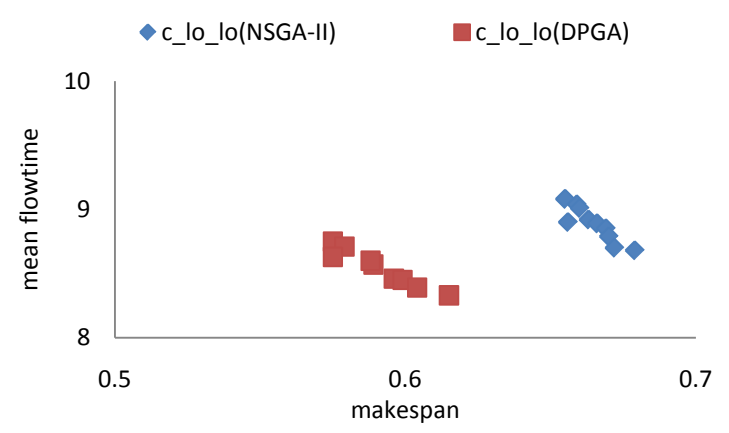

(a)

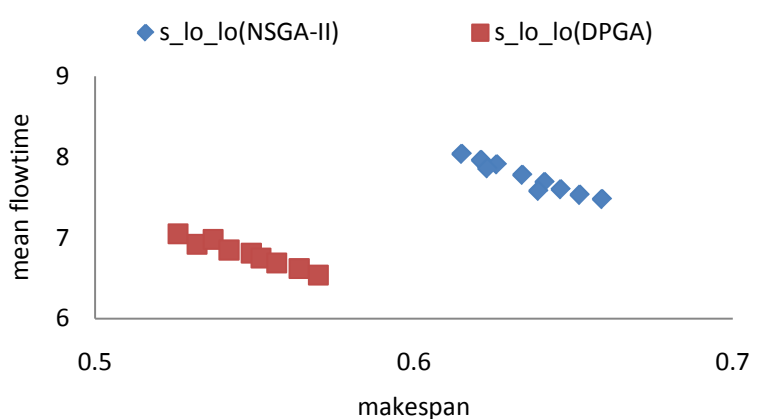

(a)

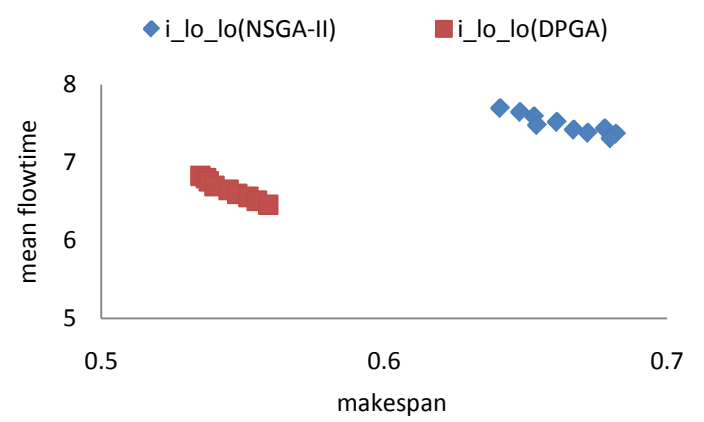

(b)

Figure 4. NSGA-II with MapReduce and DPGA with MapReduce comparison for low task, low processor heterogeneity. (a) Consistent; (b) Semi consistent; (c) Inconsistent.

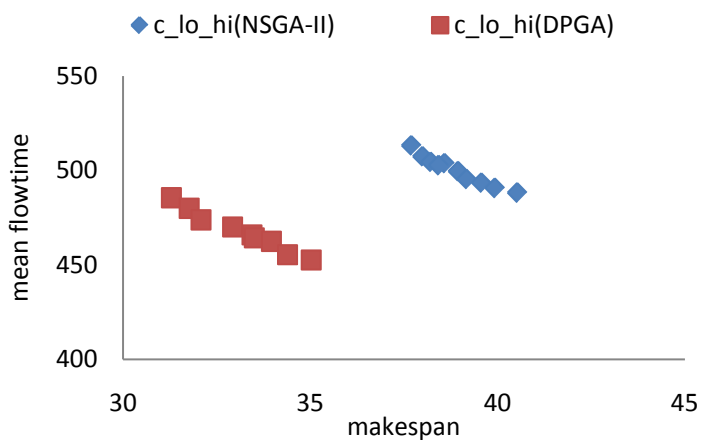

(a)

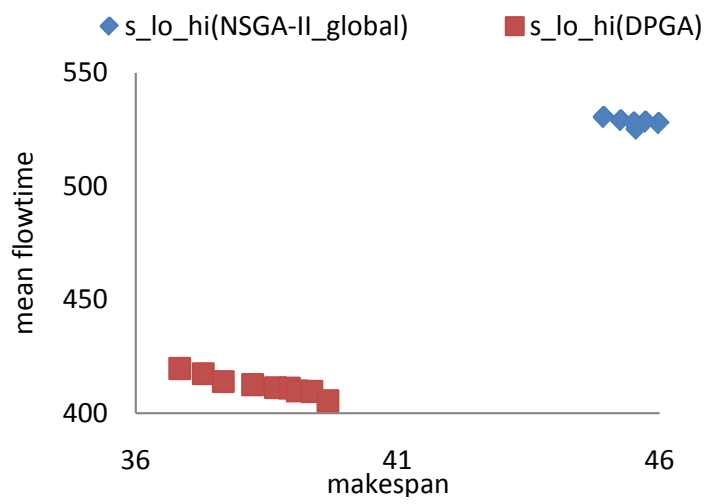

(b)

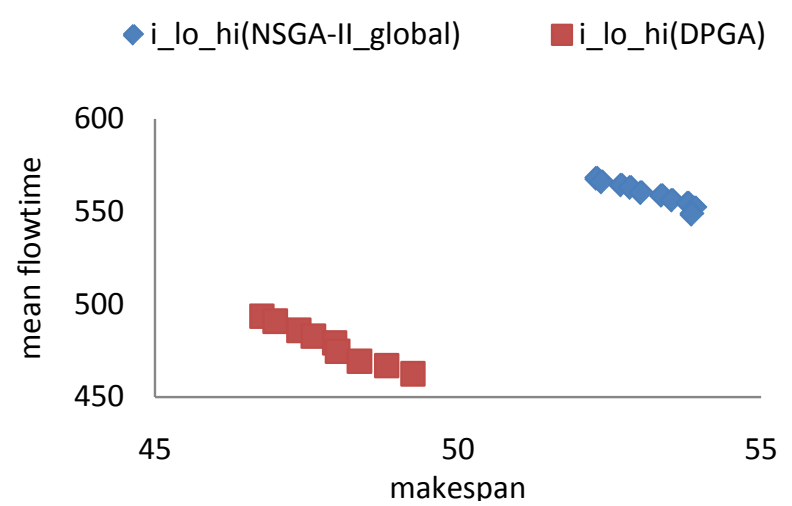

(c)

Figure 5. NSGA-II with MapReduce and DPGA with MapReduce comparison for low task, high processor heterogeneity. (a) Consistent; (b) Semi consistent; (c) Inconsistent. 


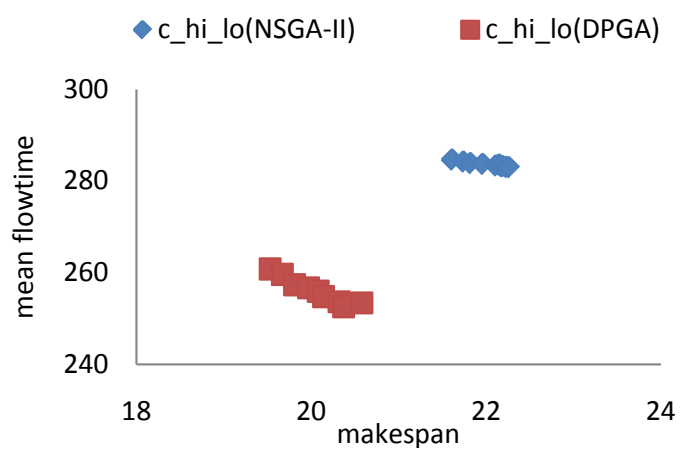

(a)

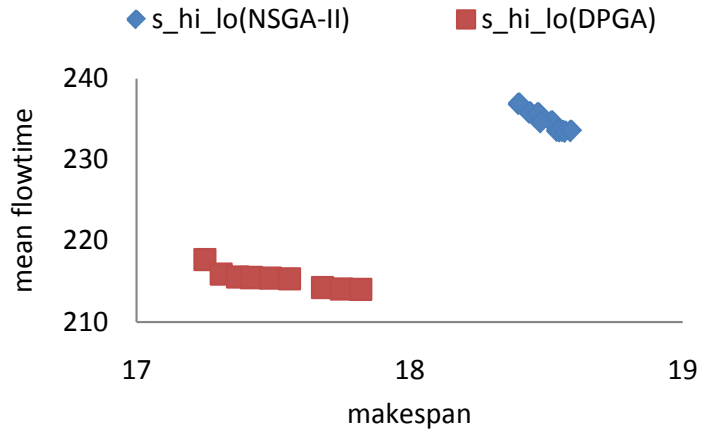

(b)

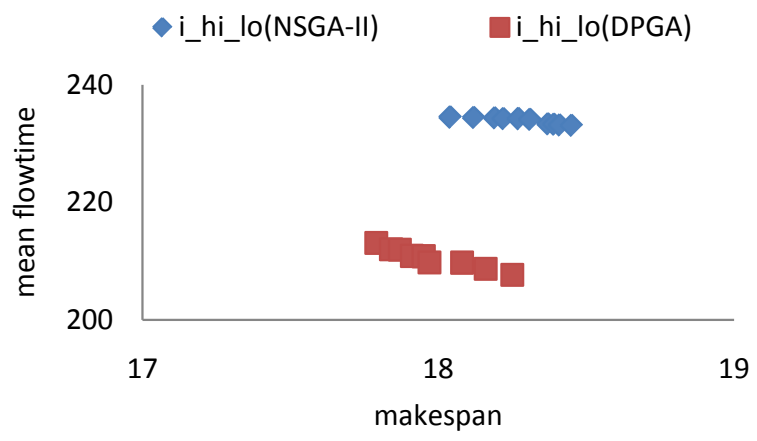

(c)

Figure 6. NSGA-II with MapReduce and DPGA with MapReduce comparison for high task, low processor heterogeneity. (a) Consistent; (b) Semi consistent; (c) Inconsistent.

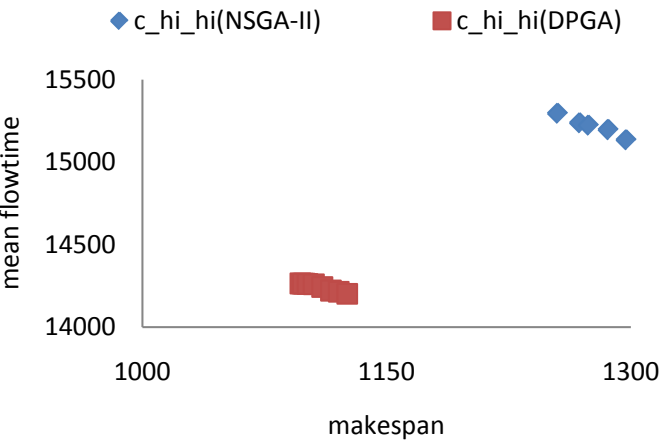

(a)

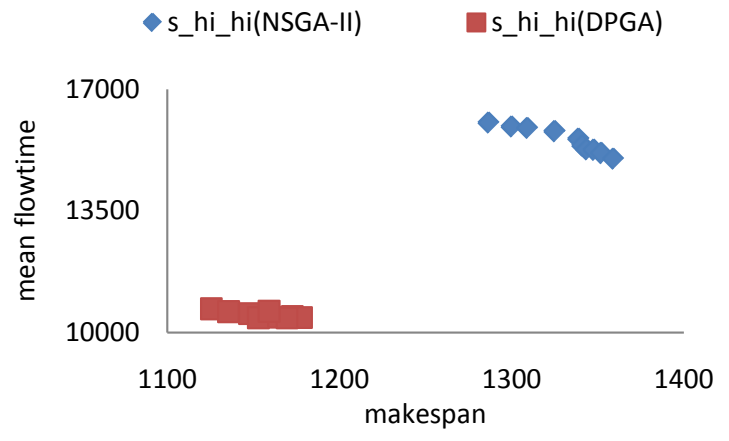

(b)

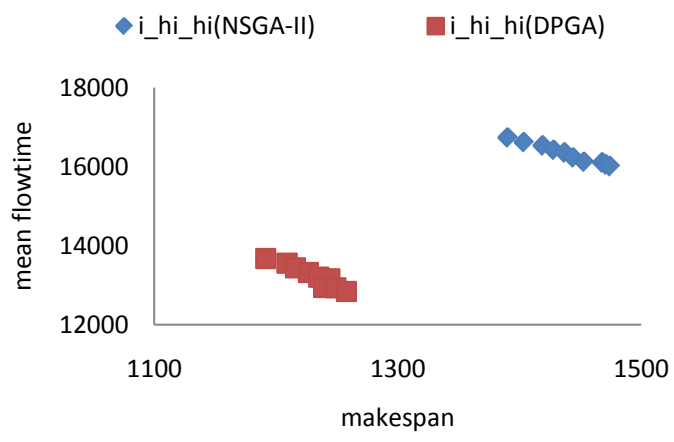

(c)

Figure 7. NSGA-II with MapReduce and DPGA with MapReduce comparison for high task, high processor heterogeneity. (a) Consistent; (b) Semi consistent; (c) Inconsistent. 
population and number of iterations. Figures 4-7 show that DPGA with MapReduce model generates better makespan and mean flowtime for all consistency type, task and processor heterogeneity.

\subsection{Performance Comparison of NSGA-II and DPGA}

The Pareto optimal solution set obtained by NSGA-II, DPGA satisfy different objectives to some extend [26]. A fuzzy based technique is used to select best compromise solution from the attained non-dominated set of solutions [27]. The fuzzy sets are defined using triangular membership function. Consider $\mathrm{f}^{\max }$ and $\mathrm{f}^{\min }$ are maximum and minimum values of each objective function, solution in the $\mathrm{k}^{\text {th }}$ objective function of a Pareto set $\mathrm{f}_{\mathrm{k}}$ is described by a membership function $\mu_{\mathrm{k}}$ illustrated as,

$$
\mu_{k}= \begin{cases}1, & f_{k} \leq f_{\min } \\ \frac{f_{k}^{\max }-f_{k}}{f_{k}^{\max }-f_{k}^{\min }}, & f_{k}^{\min }<f_{k}<f_{k}^{\max } \\ 0, & f_{k} \geq f_{\max }\end{cases}
$$

The value of membership function indicates how far a non-dominated solution has satisfied the objective. In order to measure the performance of each solution to satisfy the objective, the sum of membership function values $\mu_{\mathrm{k}}$ is computed, where $k=1,2, \cdots, m$ objectives. The performance of each non-dominated solution can be rated with respect to the entire $\mathrm{N}$ non-dominated solutions by normalizing its performance over the sum of the ability of $\mathrm{N}$ non-dominated solutions as follows,

$$
\mu_{i}=\frac{\sum_{k=1}^{m} \mu_{k}^{i}}{\sum_{i=1}^{n} \sum_{k=1}^{m} \mu_{k}^{i}}
$$

where $n$ is the amount of solution and $m$ is the amount of objective functions. The solution has the best value of $\mu_{\mathrm{i}}$ is the solution. The makespan and mean flow time value for the finet adjustment solution attained is listed in Table 2. The percentage of reduction in makespan and mean flow time of DPGA with MapReduce over NSGA-II with MapReduce is calculated as,

$$
\begin{aligned}
& \text { percentage of makespan reduction }=1-\frac{\text { makespan }_{\mathrm{DPGA}}}{\text { makespan }_{\mathrm{NSGA}-\mathrm{II}}} \times 100 \\
& \text { percentage of mean flowtime reduction }=1-\frac{\text { mean flowtime }_{\text {DPGA }}}{\text { mean }_{\text {flowtime }}} \times 100
\end{aligned}
$$

The makespan and mean flow time reduction percentage is present in the Table 2. DPGA with MapReduce achieves a reduction in makespan and flowtime by $12 \%$ and $14 \%$, over the values of NSGA-II with MapReduce. Figures 4-7 also indicates DPGA with MapReduce outperforms over NSGA-II with MapReduce.

\subsection{Execution Time Comparison of NSGA-II and DPGA}

NSGA-II with MapReduce and DPGA with MapReduce is executed in single node and 4 node Hadoop cluster. The time taken by both the algorithms to find the optimal schedule is listed in Table 3. It is noted that DPGA with MapReduce has less execution time than NSGA-II with MapReduce. As the number of nodes in a Hadoop cluster is increased, the execution time of these algorithms will be reduced.

\section{Conclusion}

In distributed computing systems, allocation of tasks to the processing element with minimum amount of time is a key step for better utilization of resources. In this paper, NSGA-II with MapReduce and DPGA with MapReduce is implemented in DS environment and their makespan, mean flow time and execution time are compared. From the obtained results, it is noted that DPGA with MapReduce model achieves a reduction in makespan, mean flow time and execution time by $12 \%, 14 \%$ and $13 \%$. The simulation results also show that the execution 
Table 2. Comparison of NSGA-II with MapReduce and DPGA with MapReduce.

\begin{tabular}{rcccccc}
\hline \multirow{2}{*}{ Instances } & NSGA_II with MapReduce and Fuzzy & \multicolumn{2}{c}{ DPGA with MapReduce and Fuzzy } & $\begin{array}{c}\text { Percentage of } \\
\text { Reduction in }\end{array}$ & $\begin{array}{c}\text { Percentage of } \\
\text { Reduction in mean } \\
\text { flow time by DPGA }\end{array}$ \\
\cline { 2 - 5 } ma_lo_lo & 6546.28 & 90838.43 & 5748.18 & 85539.56 & 12.19 & 5.83 \\
co_lo_hi & 376851.95 & 5133760.26 & 313263.46 & 4354684.62 & 16.87 & 15.18 \\
co_hi_lo & 216064.67 & 2846845.19 & 194562.67 & 2605329.13 & 9.95 & 8.48 \\
co_hi_hi & 12548739.42 & 152960763.32 & 10965632.48 & 142659273.5 & 12.62 & 6.73 \\
sc_lo_lo & 6145.27 & 80376.11 & 5259.03 & 70540.74 & 14.42 & 12.24 \\
sc_lo_hi & 449285.18 & 5304352.69 & 388432.8 & 4146759.85 & 13.54 & 21.82 \\
sc_hi_lo & 184273.52 & 2368543.50 & 170501.59 & 2175234.38 & 7.47 & 8.16 \\
sc_hi_hi & 12864789.63 & 160436981.84 & 11259642.43 & 106847994.9 & 12.48 & 33.40 \\
ic_lo_lo & 6389.37 & 76943.33 & 5346.35 & 68259.43 & 16.32 & 11.29 \\
ic_lo_hi & 522843.21 & 5679384.21 & 467899.59 & 4938345.23 & 10.51 & 13.05 \\
ic_hi_lo & 180437.35 & 2344622.15 & 171243.62 & 2132541.59 & 5.10 & 9.05 \\
ic_hi_hi & 13901746.88 & 167435439.5 & 11913592.13 & 138565274.3 & 14.30 & 17.24 \\
\hline
\end{tabular}

Table 3. Comparison of execution time.

\begin{tabular}{|c|c|c|c|}
\hline Methods & NSGA-II with MapReduce & DPGA with MapReduce & Percentage of reduction \\
\hline Sequential (Single node) & $34.68 \mathrm{Sec}$ & $31.93 \mathrm{Sec}$ & 7.93 \\
\hline $\begin{array}{l}\text { Parallelism with MapReduce } \\
\text { (4 node Cluster) }\end{array}$ & $31.714 \mathrm{Sec}$ & $27.64 \mathrm{Sec}$ & 12.85 \\
\hline
\end{tabular}

time of these algorithms will be reduced, while increasing the number of nodes in a Hadoop cluster. Future work could be extended for implementing a scheduler by using all the evolutionary kind of algorithms with the MapReduce model to execute its parallel without coordination issue.

\section{References}

[1] Braun, T.D., Siegel, H.J, Beck, N., Boloni, L.L., Maheswaran, M., Reuther, A.I., Robertson, J.P., Theys, M.D. and Yao, B. (2001) Comparison of Eleven Static Heuristics for Mapping a Class of Independent Tasks onto Heterogeneous Distributed Computing Systems. Journal of Parallel and Distributed Computing, 61, 810-837. http://dx.doi.org/10.1006/jpdc.2000.1714

[2] Subashini, G. and Bhuvaneswari, M.C. (2011) Non Dominated Particle Swarm Optimization for Scheduling Independent Tasks on Heterogeneous Distributed Environments. International Journal of Advances in Soft Computing and Its Applications, 3, 1.

[3] Kaiwartya, O., Prakash, S., Abdullah, A.H. and Hassan, A.N. (2015) Minimizing Energy Consumption in Scheduling of Dependent Tasks Using Genetic Algorithm in Computational Grid. KSII Transactions on Internet and Information Systems, 9, 8.

[4] Abraham, A., Liu, H., Zhang, W. and Chang, T.G. (2006) Scheduling Jobs on Computational Grids Using Fuzzy Particle Swarm Algorithm. Springer-Verlag, Berlin, Heidelberg, 500-507. http://dx.doi.org/10.1007/11893004_65

[5] Deb, K. (2002) Multi-Objective Optimization Using Evolutionary Algorithms. Wiley Publications.

[6] Osycaka, A. and Kundu, S. (1995) A New Method to Solve Generalized Multicriteria Optimization Problems Using the Simple Genetic Algorithm. Structural Optimization, 10, 94-99. http://dx.doi.org/10.1007/BF01743536

[7] Wang, F., Qiu, J., Yang, J., Dong, B., Li, X. and Li, Y. (2009) Hadoop High Availability through Metadata Replication. In: Cloud, D.B., Ed., Proceedings of the First International Workshop on Cloud Data Management. http://dx.doi.org/10.1145/1651263.1651271

[8] Munir, E.U., Li, J.-Z., Shi, S.-F. and Rasool, Q. (2007) Performance Analysis of Task Scheduling Heuristics in Grid. 
ICMLC'07: Proceedings of the International Conference on Machine Learning and Cybernetics, 6, 3093-3098. http://dx.doi.org/10.1109/icmlc.2007.4370679

[9] Maheswaran. M., Ali, S., Siegel, H.J., Hensgen, D. and Freund, R.F. (1999) Dynamic Mapping of a Class of Independent Tasks onto Heterogeneous Computing Systems. Journal of Parallel and Distributed Computing, 59, 107-131. http://dx.doi.org/10.1006/jpdc.1999.1581

[10] Freund, R.F., Gherrity, M., Ambrosius, S., Campbell, M., Halderman, M., Hensgen, D., Keith, T., Kussow, M., Lima, J.D., Mirabile, F., Moore, L., Rust, B. and Siegel, H.J. (1998) Scheduling Resources in Multiuser, Heterogeneous, Computing Environments with SmartNet. 7th IEEE Heterogeneous Computing Workshop, 184-199.

[11] Abraham, A., Buyya, R. and Nath, B. (2000) Nature's Heuristics for Scheduling Jobs on Computational Grids. The 8th IEEE International Conference on Advanced Computing and Communications (ADCOM 2000), India, 14-16.

[12] Kang, Q.M. and He, H. (2011) A Novel Discrete Particle Swarm Optimization Algorithm for Meta-Task Assignment in Heterogeneous Computing Systems. Microprocessors and Microsystems, 35, 10-17. http://dx.doi.org/10.1016/j.micpro.2010.11.001

[13] Pacini, E., Mateos, C. and Garino, C.G. (2015) Balancing Throughput and Response Time in Online Scientific Clouds via Ant Colony Optimization. Advances in Engineering Software, 84, 31-47. http://dx.doi.org/10.1016/j.advengsoft.2015.01.005

[14] Hossein, S., Doulabi, H., Avazbeigi, M., Arab, S. and Davoudpour, H. (2012) An Effective Hybrid Simulated Annealing and Two Mixed Integer Linear Formulations for Just-in-Time Open Shop Scheduling Problem. The International Journal of Advanced Manufacturing Technology, 59, 1143-1155.

[15] Izakian, H., Abraham, A. and Snasel, V. (2009) Comparison of Heuristics for Scheduling Independent Tasks on Heterogeneous Distributed Environments. IEEE Control Systems Magazine, 1, 8-12.

[16] Abraham, A., Liu, H., Grosan, C. and Xhafa, F. (2008) Nature Inspired Meta-Heuristics for Grid Scheduling: Single and Multi-Objective Optimization Approaches. In: Xhafa, F. and Abraham, A., Eds., Metaheuristics for Scheduling in Distributed Computing Environments, Springer Verlog, Berlin, 247-272. http://dx.doi.org/10.1007/978-3-540-69277-5_9

[17] Carretero, J., Xhafa, F. and Abraham, A. (2007) Genetic Algorithm Based Schedulers for Grid Computing Systems. International Journal of Innovative Computing, Information and Control, 3, 1-19.

[18] Lim, D., Ong, Y.-S., Jin, Y., Sendhoff, B. and Lee, B.-S. (2007) Efficient Hierarchical Parallel Genetic Algorithms Using Grid Computing. Future Generation Computing Systems, 23, 658-670. http://dx.doi.org/10.1016/j.future.2006.10.008

[19] Durillo, J.J., Nebro, A.J., Luna, F. and Alba, E. (2008) A Study of Master-Slave Approaches to Parallelize NSGA-II. IEEE International Symposium on Parallel and Distributed, Miami, 14-18 April 2008, 1-8. http://dx.doi.org/10.1109/IPDPS.2008.4536375

[20] Subhashini, G. and Bhuvaneswari, M.C. (2010) A Fast and Elitist Bi-Objective Evolutionary Algorithm for Scheduling Independent Tasks on Heterogeneous Systems. ICTACT, Journal on Soft Computing, 1, 9-17.

[21] Rajeswari, D. and Jawahar Senthilkumar, V. (2015) Multiprocessor Scheduling and Performance Evaluation Using Elitist Non-Dominated Sorting Genetic Algorithm for Independent Task. International Journal on Computational Science \& Applications, 5, 49-59.

[22] Deb, K., Pratap, A., Agarwal, S. and Meyarivan, T. (2002) A Fast Elitist Multiobjective Genetic Algorithm: NSGA-II. IEEE Transactions on Evolutionary Computation, 6, 182-197. http://dx.doi.org/10.1109/4235.996017

[23] Rajeswari, D. and Jawahar Senthilkumar, V. (2015) Multiprocessor Scheduling and Performance Evaluation Using Distance-Based Pareto Genetic Algorithm for Independent Task. International Journal of Applied Engineering Research, 10, 33-38.

[24] Dhole Poonam, B. and Gunjal Baisa, L. (2013) Survey Paper on Traditional Hadoop and Pipelined Map Reduce. International Journal of Computational Engineering Research, 3, 32-36.

[25] http://hadoop.apache.org/docs/r1.2.1/mapred_tutorial.html

[26] Niimura, T. and Nakashima, T. (2003) Multiobjective Tradeoff Analysis of Deregulated Electricity Transactions. International Journal of Electrical Power \& Energy Systems, 25, 179-185. http://dx.doi.org/10.1016/S0142-0615(02)00076-5

[27] Guzek, M., Pecero, J.E., Dorronsoro, B. and Bouvry, P. (2014) Multi-Objective Evolutionary Algorithms for Energy-Aware Scheduling on Distributed Computing Systems. Applied Soft Computing, 24, 432-446. http://dx.doi.org/10.1016/j.asoc.2014.07.010 